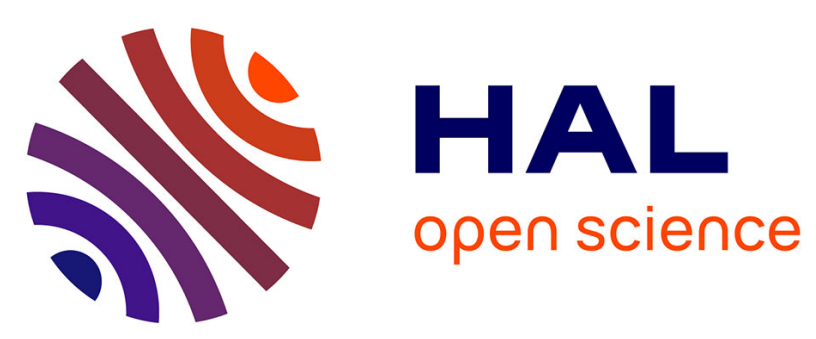

\title{
Dynamic Parameters Identification of an Industrial Robot: a constrained nonlinear WLS approach
}

\author{
Abdelkrim Bahloul, Sami Tliba, Yacine Chitour
}

\section{To cite this version:}

Abdelkrim Bahloul, Sami Tliba, Yacine Chitour. Dynamic Parameters Identification of an Industrial Robot: a constrained nonlinear WLS approach. IEEE MED'18: The 26th Mediterranean Conference on Control and Automation, Jun 2018, Zadar, Croatia. pp.21-26, 10.1109/MED.2018.8442630 . hal01866334

\section{HAL Id: hal-01866334 \\ https://hal.science/hal-01866334}

Submitted on 3 Sep 2018

HAL is a multi-disciplinary open access archive for the deposit and dissemination of scientific research documents, whether they are published or not. The documents may come from teaching and research institutions in France or abroad, or from public or private research centers.
L'archive ouverte pluridisciplinaire HAL, est destinée au dépôt et à la diffusion de documents scientifiques de niveau recherche, publiés ou non, émanant des établissements d'enseignement et de recherche français ou étrangers, des laboratoires publics ou privés. 


\title{
Dynamic Parameters Identification of an Industrial Robot: a constrained nonlinear WLS approach
}

\author{
Authors: Abdelkrim Bahloul, Sami Tliba and Yacine Chitour
}

A. Bahloul, S. Tliba and Y. Chitour are with Laboratoire des Signaux et Systèmes UMR8506, Univ Paris-Sud, CNRS, CentraleSupelec, Université Paris-Saclay, 3 rue Joliot Curie, 91192 Gif-sur-Yvette, France (corresponding author e-mail: sami.tliba@u-psud.fr).

Université Paris-Sud XI,

U.F.R. des Sciences d'Orsay

Layout by Sami TLIBA, (C) Aout 2018
UMR : Laboratoire des Signaux \& Systèmes

Projet : Robotic comanipulation

Type : Postprint of "2018 IEEE Mediteranean Conference on Control and Automation" 


\begin{abstract}
This paper brings an identified model for a 6 degrees of freedom (dof) industrial robot, the Denso VP-6242G robot, with an end-effector composed of a spherical handle, fixed on a force sensor. This robot is intended to experiments in the field of Physical Human-Robot Interactions (PHRIs), for co-manipulation purposes. The control algorithms that are necessary to achieve a good PHRI, require a good knowledge of the robot dynamical model, especially the inertia matrix which should be positive definite whatever the configuration of the robot. However, most of industrial robots are supplied without any datasheet containing the inertial parameters nor Computer-Aided-Design (CAD) model. Hence, we propose to apply an identification procedure to experimental data, based on the Inverse Dynamic model Identification Method (IDIM). To ensure the positive definiteness of the inertia matrix, the used optimization step addresses the problem of nonlinear Weighted Least Squares (WLS), derived from the mathematical formulation of the identification problem, under a set of nonlinear constraints in the parameters. A validation step permits to check the efficiency of this approach for the Denso robot.
\end{abstract}

keywords: Industrial Robot; Closed-Loop Identification; Constrained Optimization.

\title{
1 Introduction
}

In control engineering field, most of the advanced nonlinear control techniques require the knowledge of the system's dynamical model. It is the case for many control problems concerning industrial robot manipulators, for which good trajectory tracking has to be carried out. Many works about Physical Human-Robot Interactions (PHRI) rely on the principle of trajectory tracking. This is for example the case in [Jlassi et al., 2014] where the authors have addressed the co-manipulation problem which consists in achieving a master-slave relationship between an industrial robot and a human operator (HO). A dynamical model contains kinematic and dynamic parameters. The kinematic parameters are known as the parameters of the Modified Denavit-Hartenberg convention (MDH) [Khosla and Kanade, 1985]. It is not difficult to extract these latter based on the robot datasheet provided by the manufacturers. However, dynamic parameters, which are inertial and friction parameters, are rarely available in such datasheet. Moreover, it is hard, say impossible, to get an accurate Computer-Aided-Design (CAD) model in order to obtain a good approximation of some inertial parameters. Then, the only way to get a good dynamical model is the use of identification techniques applied to experimental data, that can be found in [Walter and Pronzato, 1997]. This topic has received a great attention by the robotics community during the last thirty years. Several important issues have been addressed by researchers. A non exhaustive list of issues can be found in [Swevers et al., 2007]. Among them, the obtaining of a dynamical model is of utmost importance because it determines the number of parameters to be estimated. This issue has lead the researchers to distinguish between the notions of link inertial parameters and base parameters, as introduced in [Gautier and Khalil, 1990, Gautier, 1990]. Basically, the base parameters are a kind of parameters' regrouping within the motion equations, that allows to formulate these motion equations as a linear relation in these parameters. This turns out to be useful when deriving an algorithm of parameters' estimation. Another issue concerns the experimental design, including the robot excitation [Jin and Gans, 2015, Gautier and Khalil, 1991]. Most of people suggested a data acquisition in closed-loop, using a jointposition control with a simple proportional controller designed to ensure a desired trajectory tracking. Even if the closedloop feature creates some correlations between the noise affecting the measurements and the other signals within the closed-loop structure, this approach turns out to be quite efficient in the parameters' estimation if the measured signals are well post-processed with a suitable filtering [Janot et al., 2014, Brunot et al., 2017]. The desired trajectories are almost always designed as periodic functions, corresponding to a sum of sinusoids with appropriate features, while guaranteeing an acceptable Signal-to-Noise-Ratio (SNR) during the acquisition. This allows the inputs to meet the expected excitation properties for the parameters' identifiability. Finally, the estimated parameters' computation method is closely related to the kind of model used for identification. The Inverse Dynamic model Identification Method (IDIM) is the most popular one since it provides an equivalent formulation of the torques that is linear in the base parameters. Hence, the Least 
Squares (LS) approach can easily be performed [Swevers et al., 2007, Jin and Gans, 2015]. Most of works have rather used the Weighted Least Squares (WLS) technique to prevent the effects of inaccurate data. To this end, the data are averaged over one period and weighted by the covariance matrix of the torque measurements, computed by taking into account all the recorded periods. Nevertheless, there is no guarantee that the estimated parameters lead to a positive definite inertial matrix [Yoshida and Khalil, 2000].

In this paper, we propose to combine a nonlinear WLS estimation technique under a set of nonlinear constraints in the dynamic parameters, that ensures the inertia matrix to be positive definite. The paper is organized as follows, Section 2 introduces the basics on robot identification, and explains the constrained nonlinear WLS approach. Then, Section 3 proposes the application to the Denso VP-6242G robot. Finally, some conclusions are proposed in Section 4.

\section{Basics on robot identification}

Consider the general case of a rigid robot having $n$ revolute joints, with $n$ serial links and a fixed base. The kinematic parameters are assumed to be well known. The dynamic parameters stands for the inertial parameters of the links as defined in [Yoshida and Khalil, 2000], and the usual Coulomb \& viscous friction coefficients as indicated in [Swevers et al., 2007]. A parametric equation of motion in these parameters can be formulated, in continuous time, as:

$$
M(q, \theta) \ddot{q}+N(q, \dot{q}, \theta)=\tau
$$

where $q, \dot{q}$ and $\ddot{q}$ denote the $(n \times 1)$ vectors of joint positions, velocities and accelerations respectively, called kinematic joint variables; $\tau$ is the $(n \times 1)$ vector of the joint torques; $M(q, \theta)$ denotes the $(n \times n)$ inertia matrix which is symmetric and positive definite; $N(q, \dot{q}, \theta)$ is the $(n \times 1)$ vector that gathers the Coriolis, centrifugal, gravity and friction torques. Both terms depend on the dynamic parameters' vector denoted $\theta$, of dimension $\left(n_{\theta} \times 1\right)$, in a linear manner. It is assumed that all the components of $q$ and $\tau$ are measured with noisy sensors.

\subsection{Choice of the parameters for the IDIM approach}

The IDIM consists in rewriting the left side of (1) as a linear relation in $\theta$ as follows:

$$
\tau=D(q, \dot{q}, \ddot{q}) \theta
$$

where $D$ is a $\left(n \times n_{\theta}\right)$ matrix that only depends on the kinematic joint variables of the robot, called the measurement matrix [An et al., 1985]. By using the same notations as in [Khalil and Creusot, 1997] for the link $i$, we can define the following dynamic parameters:

- $x x_{i}, x y_{i}, x z_{i}, y y_{i}, y z_{i}, z z_{i}$ are the components of the inertia tensor matrix ${ }^{i} I_{i}$ around the origin $O_{i}$ of the link frame $R_{i}$;

- $m x_{i}, m y_{i}, m z_{i}$ are the first moments;

- $m_{i}$ is the mass of the link;

- $F s_{i}, F v_{i}$ are the Coulomb and viscous friction coefficients of a friction model linear in these parameters.

The above parameters are gathered in the following vector

$$
\begin{aligned}
\theta_{i}^{T}=\left[\begin{array}{lllllll}
x x_{i} & x y_{i} & x z_{i} & y y_{i} & y z_{i} & z z_{i} \\
& m x_{i} & m y_{i} & m z_{i} & m_{i} & F s_{i} & F v_{i}
\end{array}\right] .
\end{aligned}
$$


where ${ }^{T}$ denotes the transpose operator. The first ten parameters of $\theta_{i}$ are called the inertial parameters for the link $i$, as indicated in [Yoshida and Khalil, 2000]. Then, $\theta$ is built by concatenating the $\theta_{i}(i=1, \ldots, n)$.

In [Gautier and Khalil, 1990, Gautier, 1990], the authors have proposed to determine which are the parameters that have no influence on the dynamics of the robot. They derived some rules for the regrouping of the inertial parameters and the corresponding relationships. The regrouped inertial parameters are called minimum inertial parameters. The new parameters' vector is called the base parameter vector, denoted by $\theta_{B}$, and contains $n_{\theta_{B}}$ components. Relation (2) can thus be rewritten as

$$
\tau=D_{B}(q, \dot{q}, \ddot{q}) \theta_{B}
$$

where $D_{B}$ is a $\left(n \times n_{\theta_{B}}\right)$ matrix, with $n_{\theta_{B}}<n_{\theta}$.

\subsection{Closed-loop data acquisition and excitation signals}

For the sake of keeping the robot integrity, it is better to excite the robot while mastering its configuration at each sampling time, in order to avoid self collisions or collisions of the robot with its environment. Hence, a joint position feedback control with a simple proportional controller, with suitable adjusted gain, is performed to follow a given trajectory in joint space that meets a number of desired requirements. In identification, the choice of the excitation signal is important, because it should "excites all the parameters" to identify. A signal with this feature is called a Persistent Excitation Signal (PES). A PES used to identify $n_{\theta_{B}}$ parameters must contains at least $n_{\theta_{B}}$ sinusoids of different frequencies [Gautier and Khalil, 1988], [Gautier and Khalil, 1991], [Lennart, 1971]. Moreover, the desired trajectory should be designed so that it is periodic during a long time range in order to ease the statistical analysis of the results [Swevers et al., 2007], each period being built to be a PES and the number of periods should be greater than $n$. Finally, the amplitudes of the PES should be within an allowable range of motion, in order to comply with the robot safety limits, and it should maximize the Signal-to-Noise-Ratio (SNR) for each measured output signal needed for the robot identification. One can easily design such PES with the requested properties and uses them for the robot identification as desired trajectories for the joint positions. If the proportional gains of the position feedback control are high enough, then the tracking errors between the measured and the desired joint positions become small enough to ensure that the measured positions will have properties close to the ones of the desired trajectories.

\subsection{Signal processing}

In general, the measured (joint torques and positions) and computed (joint velocities and accelerations) data are noisy. These data can not be directly used in identification because of the closed-loop feedback control that might create correlations between the noises and the signals of the closed-loop system. This can introduce some unknown biases in the values of the identified parameters [Brunot et al., 2017]. To avoid this undesirable effect, the data are filtered before being used by the estimation algorithms. This is done off-line thanks to a low-pass filter with a unit gain at low frequencies and a frequency cut-off set a decade after the highest existing frequency in the desired trajectories. In practice, a forward plus backward filtering of the noisy data is performed in order to get a zero-phase filtering of these data. Furthermore, since the applied excitation signals contain many periods, an average period of each signal is computed over all the available periods in order to reduce the computational burden of the estimation algorithm. Given a periodic signal in discrete time, denoted by $x(k)$, with $P$ full periods and $K$ samples per period. The average period of $x(k)$, denoted by $\bar{x}(k)$, is obtained through the relation

$$
\bar{x}(k)=\frac{1}{P} \sum_{p=1}^{p=P} x(k+K(p-1)) .
$$

Finally, it may sometimes be useful to undersample the data if the sampling frequency is too high compared to the frequency bandwidth of interest. 
Let $\hat{q}$ and $\hat{\tau}$ be the joint positions and torques after applying the whole signal processing described before; $\dot{\hat{q}}$ and $\ddot{\hat{q}}$ are the resulting joint velocities and accelerations, suitably computed by numerical differentiation. The data are assumed to check the following relation at sample time $k$, derived from (4)

$$
\hat{\tau}(k)=D_{B}(\hat{q}(k), \dot{\hat{q}}(k), \ddot{\hat{q}}(k)) \theta_{B}+\rho(k),
$$

where $\rho$ is an $(n \times 1)$ vector of error terms, gathering the noises and the unmodelled dynamics. It is assumed to have a zero-mean, and uncorrelated samples. The sets of $N$ samples retained after the whole signal processing described above are used to define:

$$
Y:=\left[\begin{array}{c}
\hat{\tau}(1) \\
\vdots \\
\hat{\tau}(N)
\end{array}\right], W:=\left[\begin{array}{c}
D_{B}(\hat{q}(1), \dot{\hat{q}}(1), \ddot{\hat{q}}(1)) \\
\vdots \\
D_{B}(\hat{q}(N), \dot{\hat{q}}(N), \ddot{\hat{q}}(N))
\end{array}\right], R:=\left[\begin{array}{c}
\rho(1) \\
\vdots \\
\rho(N)
\end{array}\right],
$$

where $Y, R$ are $(n N \times 1)$ vectors, and $W$ is $\left(n N \times n_{\theta_{B}}\right)$ matrix. Then, (6) leads to

$$
Y=W \theta_{B}+R,
$$

which is an overdeterminated set of linear equations in the unknown parameters in $\theta_{B}$.

The conditioning number associated with the regressor matrix $W$ allows to measure the sensitivity of the solution $\theta_{B}$ to the noises affecting the measurements of $\tau, q$ and eventually $\dot{q}$ and $\ddot{q}$. Therefore, the post-processed data should reduce the conditioning number, to ensure the convergence of the identification process towards an unbiased vector of parameters [Gautier and Khalil, 1991, Janot et al., 2014].

\subsection{Estimation of the base parameter vector}

For a full rank regressor matrix $W$, and a zero-mean additive independent Gaussian noise for $R$, an estimation $\hat{\theta}_{B}$ of $\theta_{B}$ can be obtained by solving the following WLS minimization problem [Swevers et al., 2007]:

$$
\hat{\theta}_{B}=\arg \min _{\theta_{B}}\left\|W^{T} \Sigma^{-1 / 2}\left(Y-W \theta_{B}\right)\right\|^{2}
$$

whose optimal solution is given by

$$
\hat{\theta}_{B}=C W^{T} \Sigma^{-1 / 2} Y,
$$

where $\Sigma$ denotes the covariance matrix of the actuator torque data and $C:=\left(W^{T} \Sigma^{-1 / 2} W\right)^{-1}$ is the covariance matrix of the estimated parameters' vector. The matrix $\Sigma$ is a block diagonal matrix composed of $N$ matrices of dimension $(n \times n)$ and denoted $\Sigma_{k}$, corresponding to the covariance matrix of the actuator torques computed at the sample time $k$. Its expression is given by:

$$
\Sigma_{k}=\frac{1}{(P-1)} \sum_{p=1}^{P}\left(T_{p}(k)-\hat{\tau}(k)\right)\left(T_{p}(k)-\hat{\tau}(k)\right)^{T},
$$

where $T_{p}(k)$ stands for the $p^{\text {th }}$ period at the sample time $k$ of the torque measurements $\tau$, on which has been applied only the filtering and the decimation steps. The computation of $\hat{\theta}_{B}$ using the relation (10) is very tractable, but this solution does not ensure that the corresponding inertial matrix is positive definite. In the sequel, we consider this additional constraint to the problem in (9), which becomes:

$$
\begin{gathered}
\hat{\theta}_{B}=\underset{\theta_{B}}{\arg \min _{1}}\left\|W^{T} \Sigma^{-1}\left(Y-W \theta_{B}\right)\right\|^{2} \\
\text { such that } \quad M\left(q, \hat{\theta}_{B}\right)>0 \quad \forall q .
\end{gathered}
$$


The problem (12) consists in minimizing the squared norm of the weighted error between the measured and computed joint torques, under the constraint that the corresponding inertia matrix is always positive definite, i.e. with positive eigenvalues, whatever the robot configuration. A sufficient condition for the inertial matrix to be positive definite for each configuration of the manipulator is given in [Yoshida and Khalil, 2000]. The authors have proved that constraints on physical parameters of the links composing the manipulator, lead to an inertia matrix which is positive definite. The link constraints in [Yoshida and Khalil, 2000] are:

$$
\left\{\begin{array}{l}
m_{i}>0, \quad i=1, \ldots, n \\
{ }^{i} I_{g_{i}}>0
\end{array}\right.
$$

where $m_{i}$ and ${ }^{i} I_{g_{i}}$ are the mass and the inertia tensor matrix around the center of mass $g_{i}$ of the link $i$. The relation between ${ }^{i} I_{i}$ and ${ }^{i} I_{g_{i}}$ is given by [Yoshida and Khalil, 2000]:

$$
{ }^{i} I_{i}={ }^{i} I_{g_{i}}+m_{i}\left(r_{i}^{T} r_{i} E_{3}-r_{i} r_{i}^{T}\right)
$$

where $r_{i}=\left[\begin{array}{lll}x_{i} & y_{i} & z_{i}\end{array}\right]^{T}$ is the vector from $O_{i}$ to $g_{i}$, and $E_{3}$ is the $(3 \times 3)$ identity matrix.

Let us denote the link inertial parameters mentioned in subsection 2.1 by $\theta_{I_{i}}$. The base parameters vector $\theta_{B}$ is a function of $\theta_{I_{i}}, F s_{i}$ and $F v_{i}(i=1, \ldots, n)$, whose expression can be derived analytically. Hence,

$$
\theta_{B}=f\left(\left\{\theta_{I_{i}}, F s_{i}, F v_{i}\right\}_{i=1, \ldots, n}\right) .
$$

Let us define the link physical parameters $\theta_{P_{i}}$ as the vector whose components are the elements of ${ }^{i} I_{g_{i}}, r_{i}$ and $m_{i}$. Some components of $\theta_{I_{i}}$ are also included in $\theta_{P_{i}}$, like $m_{i}$. However, other components of $\theta_{I_{i}}$ depend linearly (like $m r_{i}$ ) or nonlinearly (like ${ }^{i} I_{i}$ ) in the components of $\theta_{P_{i}}$. By denoting $h$ the general relation derived from (14), between the vectors $\theta_{I_{i}}$ and $\theta_{P_{i}}$, it follows:

$$
\theta_{I_{i}}=h\left(\theta_{P_{i}}\right), \quad i=1, \ldots, n
$$

Using the relations (15) and (16), the base parameters vector $\theta_{B}$ can be rewritten as a nonlinear function, denoted by $g$, of $\vartheta_{i}^{T}:=\left[\begin{array}{lll}\theta_{P_{i}}^{T} & F s_{i} & F v_{i}\end{array}\right]$ :

$$
\theta_{B}=g\left(\left\{\vartheta_{i}\right\}_{i=1, \ldots, n}\right)
$$

According to [Yoshida and Khalil, 2000], to have a positive definite inertia matrix, it is sufficient that all $m_{i}$ and ${ }^{i} I_{g_{i}}$ contained in the equation (17) satisfy the condition (13). However, the set of admissible $m_{i}$ and ${ }^{i} I_{g_{i}}$ is not unique. In [Yoshida and Khalil, 2000], it is called virtual parameters. The problem (12) is transformed into a Constrained WLS (CWLS) optimization new problem in the unknown parameter vector $\vartheta^{T}=\left[\begin{array}{lll}\vartheta_{1}^{T} & \cdots & \vartheta_{n}^{T}\end{array}\right]$ :

$$
\begin{aligned}
& \hat{\vartheta}=\arg \min _{\vartheta}\left\|W^{T} \Sigma^{-1}(Y-W g(\vartheta))\right\|^{2} \\
& \text { such that }\left\{\begin{array}{l}
m_{i}>0, \quad{ }^{i} I_{g_{i}}>0, \\
F s_{i}>0, \quad F v_{i}>0, \quad i=1, \ldots, n .
\end{array}\right.
\end{aligned}
$$

The constraint on the estimated inertia tensor matrix is equivalent for this last to have all its eigenvalues positive. The estimated values of $F s_{i}$ and $F v_{i}$ can only be positive, which justifies the addition of constraints on these parameters in (18). To finish the procedure, the estimated vector $\hat{\vartheta}$ is substituted into (17) to obtain the corresponding estimation of the base parameter vector $\hat{\theta}_{B}$, needed to build the estimated inertia matrix. Because of its nonlinear feature, it is worth noting that the optimization problem in (18) may contain several local minima. It should then be tackled by global optimization solvers. The global minimum seeking may be a tedious task. 


\subsection{Validation}

To check the validity of the estimated parameters by the proposed procedure, these parameters are used to reconstruct the joint torques which are compared with the measured ones. For this purpose, the post-processed kinematic joint variables are used as inputs for the inverse dynamic model in (2) with the estimated parameters. This validation step is necessary to have an information about the confidence interval of the estimated parameters, and at the same time, about the model validity.

\section{Case study : Denso VP-6242G robot}

\subsection{Dynamic identification model of the robot}

The Denso VP-6242G robot is a manipulator with six revolute joints, depicted in Fig. 1.

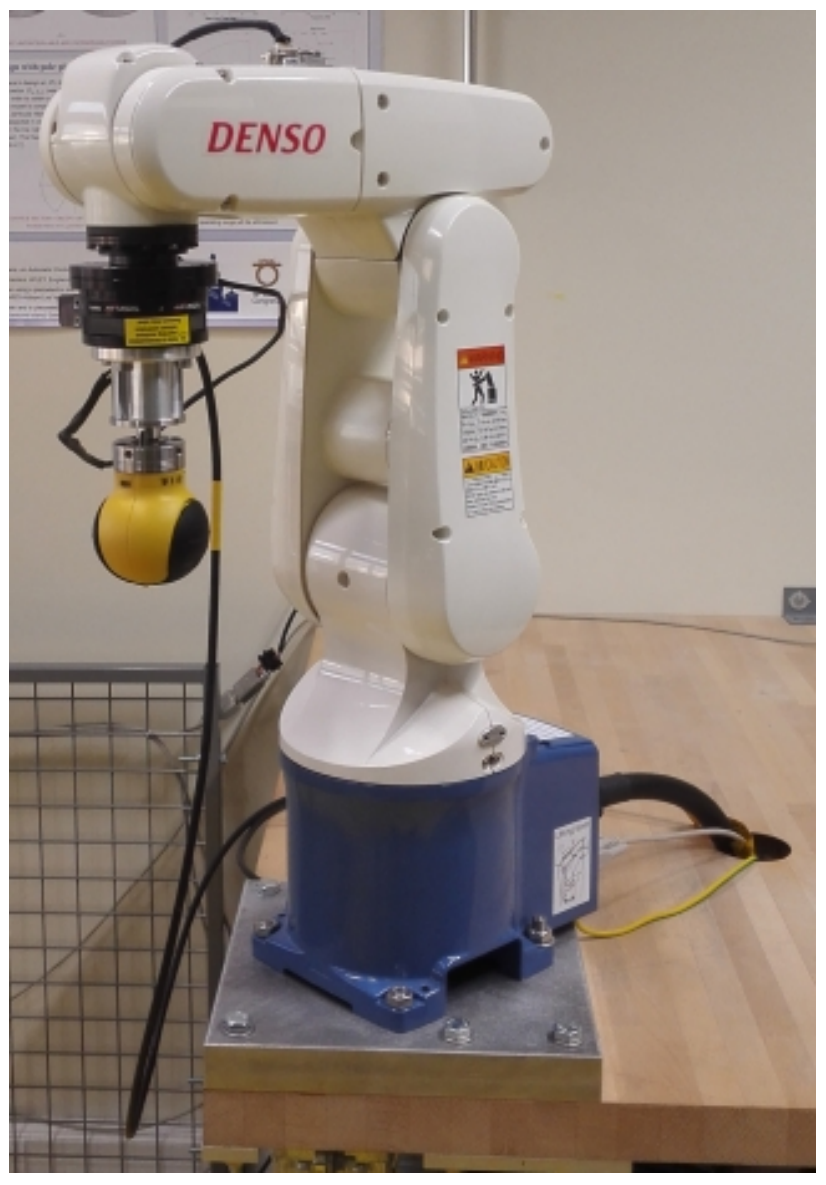

Figure 1: VP-6242G Denso robot.

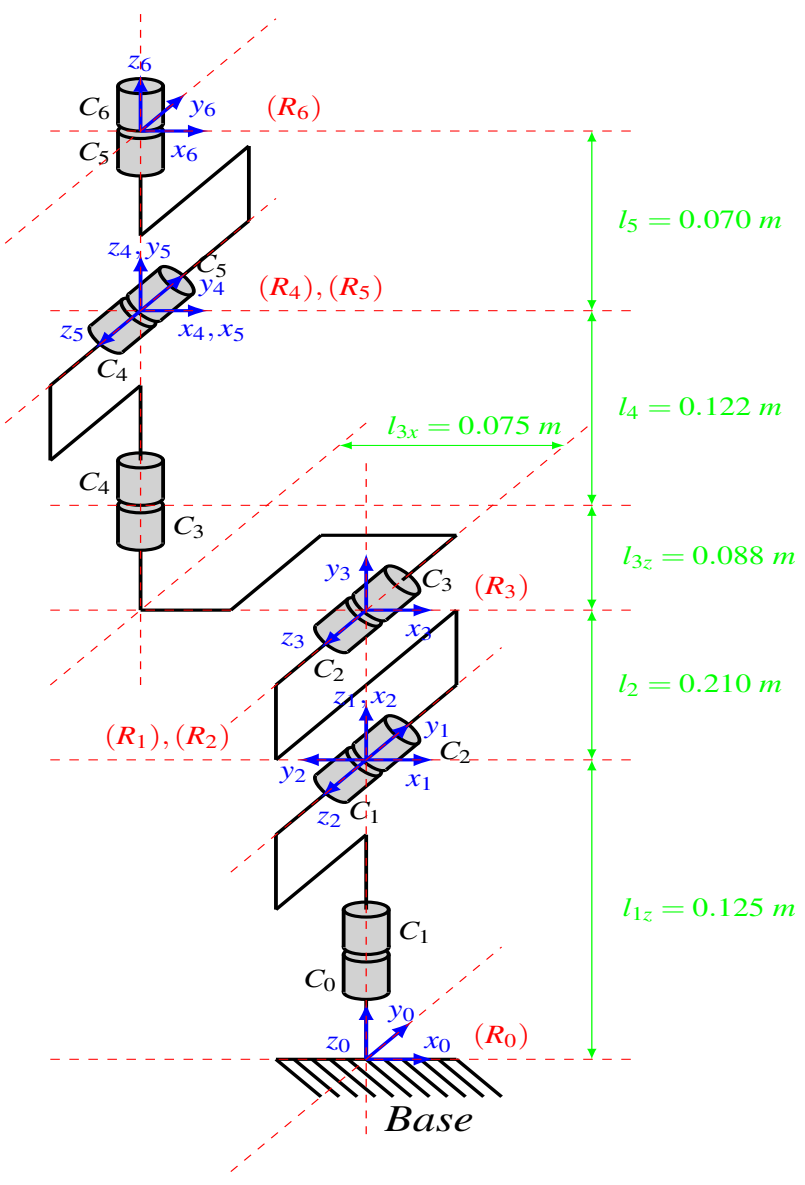

Figure 2: Robot frames for MDH convention.

A force sensor is fixed between a spherical handle, devoted to PHRI experiments, and the end-effector. The dynamic model is computed by using the parameters of the Modified Denavit-Hartenberg convention (MDH) [Khalil and Kleinfinger, 1986]. Figure 2 shows the frames attached to each joint of the robot according to this convention. Table 1 shows the MDH parameters of the Denso VP-6242G robot, extracted from Fig. 2. By using the SYMORO+ software package [Khalil and Creusot, 1997, Khalil et al., 2014] with these parameters, the dynamical model of the robot for the identification is obtained. The base parameters vector $\theta_{B}$ is of dimension $(48 \times 1)$, and the regressor matrix $D_{B}$ is of dimension $(6 \times 48)$. 
Table 1: Kinematic parameters of the Denso robot.

\begin{tabular}{c|c|c|c|c||c|c|c|c|c}
\hline \hline$i$ & $\alpha_{i}$ & $d_{i}$ & $r_{i}$ & $\theta_{i}$ & $i$ & $\alpha_{i}$ & $d_{i}$ & $r_{i}$ & $\theta_{i}$ \\
\hline \hline 1 & 0 & 0 & $l_{1 z}$ & $q_{1}$ & 4 & $-\frac{\pi}{2}$ & $-l_{3 x}$ & $l_{3 z}+l_{4}$ & $q_{4}$ \\
\hline 2 & $\frac{\pi}{2}$ & 0 & 0 & $q_{2}+\frac{\pi}{2}$ & 5 & $\frac{\pi}{2}$ & 0 & 0 & $q_{5}$ \\
\hline 3 & 0 & $l_{2}$ & 0 & $q_{3}-\frac{\pi}{2}$ & 6 & $-\frac{\pi}{2}$ & 0 & $l_{5}$ & $q_{6}$ \\
\hline \hline
\end{tabular}

\subsection{Excitation signals}

The used excitation signals, for each axis, have been designed as a sum of 55 basic sinusoids of same amplitudes, randomic phases and different frequencies. Each resulting sum was scaled to comply with its corresponding range of joint motion (see Table 2), set to keep the robot safe. The frequencies have been chosen to be integer multiples of a fundamental frequency. The number of sinusoids has been taken greater than the number of base parameters to identify in order to ensure the PES's feature [Walter and Pronzato, 1997, Lennart, 1971]. In addition, the higher frequency was constrained by the limit frequency, defined by:

$$
f_{i}=\frac{A_{\dot{q}, i}}{2 \pi A_{q, i}},
$$

where $A_{q, i}$ and $A_{\dot{q}, i}$ are the maximum allowed amplitudes for the joint position and velocity of the joint $i$. The $A_{q, i}$ are determined to avoid the collisions between the robot and its environment, and the self collisions between some parts of the robot. The $A_{\dot{q}, i}$ are derived from the actuator positioning time characteristic given in the Denso Robot datasheet, that gives a relation between positioning time and motion angle, for a given load carried by the end-effector. To enhance the estimation reliability, the periodic excitation signals have been built with 12 periods, with 380 sec per period, and a sampling time of $10^{-3} \mathrm{sec}$. The duration of one period depends on the fundamental frequency and on the limit frequency. Each initial joint position is set to the center of its corresponding joint motion range. In addition, the movement starts and stops with zero joint velocities and accelerations. For this, a phase of immobility of $4 \mathrm{sec}$ is appended at the beginning and at the end of the periodic signals. During these phases, the robot remains at the initial position. A connection phase of $5 \mathrm{sec}$ is also appended to connect the initial joint positions of the robot with the excitation signals. The total excitation time is $4578 \mathrm{sec}$. Table 2 presents the joint motion range $\left[q_{i, m}, q_{i, M}\right]$, the amplitudes $A_{q, i}$ and $A_{\dot{q}, i}$, the limit frequency $f_{i}$, and the proportional gain $K_{p_{i}}$ of the joint-position controller for each axis $J_{i}$.

Table 2: Parameters used to generate the inputs.

\begin{tabular}{c|c|c|c|c|c}
\hline \hline Axis & {$\left[q_{i, m}, q_{i, M}\right]\left[^{\circ}\right]$} & $A_{q, i}\left[^{\circ}\right]$ & $A_{\dot{q}, i}\left[^{\circ} / s\right]$ & $f_{i}[\mathrm{~Hz}]$ & $K_{p_{i}}$ \\
\hline \hline$J_{1}$ & {$[-135,+135]$} & 135 & \pm 168.7882 & 0.1989 & 1.2838 \\
\hline$J_{2}$ & {$[-45,+90]$} & 67.5 & \pm 126.4223 & 0.2981 & 1.6683 \\
\hline$J_{3}$ & {$[-90,-40]$} & 25 & \pm 168.3147 & 1.0715 & 4.4711 \\
\hline$J_{4}$ & {$[-155,+155]$} & 155 & \pm 202.1053 & 0.2075 & 1.3116 \\
\hline$J_{5}$ & {$[-90,+90]$} & 90 & \pm 205.1282 & 0.3627 & 1.7577 \\
\hline$J_{6}$ & {$[-160,+160]$} & 160 & \pm 203.6364 & 0.2025 & 1.6678 \\
\hline \hline
\end{tabular}


Table 3: Features of the motors of the Denso VP-6242G robot

\begin{tabular}{c|c|c|c|c|c|c}
\hline \hline Axis & $J_{1}$ & $J_{2}$ & $J_{3}$ & $J_{4}$ & $J_{5}$ & $J_{6}$ \\
\hline \hline$K_{c_{i}}$ & 0.38 & 0.38 & 0.22 & 0.21 & 0.21 & 0.21 \\
\hline$G_{r_{i}}$ & 120 & 160 & 120 & 100 & 100 & 100 \\
\hline \hline
\end{tabular}

\subsection{Data processing}

First, the measurements of the joint currents and positions are recorded. To obtain the joint torques, the joint currents are multiplied by their corresponding torque constants $K_{c_{i}}$ and motor gear ratios $G_{r_{i}}$, presented in Table 3 . Then, the data are filtered by using a zero-phase filtering through a low-pass Butterworth filter of order 1 with a cut-off frequency set to $10 \mathrm{~Hz}$. Joint velocities and accelerations are computed by a suitable numerical differentiation of the filtered joint positions. To reduce the noises due to numerical differentiation, the resulting velocity and acceleration signals are also filtered in the same way. In a third step, the data corresponding to the phases of immobility and connection are withdrawn in order to keep only the periodic parts of the data. The data are then averaged over the 12 periods. In a last step, since the sample frequency $\left(10^{3} \mathrm{~Hz}\right)$ is higher than the filter cutoff frequency $(10 \mathrm{~Hz})$, the data are undersampled with a factor of 10, which reduce the size of the useful data, but also the sample frequency of these data to $10^{2} \mathrm{~Hz}$. This frequency remains comfortably higher than the highest frequency contained in the filtered data (Tab. 2). Hence, the data should not be affected a lot by this decimation factor. A zoom between the instants 1200 and 1400 sec of the signals of measured joint position and torque, and calculated velocity and acceleration of the joint 1, before (blue) and after (red) filtering are shown by the figure 3 . We can observe that the noisy measurements are correctly filtered. We conclude that the choice of the filter parameters (cut-off frequency and order) is adequate. The important error in the joint acceleration signal is interpreted by a significant noise associated with this signal, as it results from a double differentiation of the joint position. We consider that these post-processed data can be used in the sequel of the identification procedure.

\subsection{Parameters estimation}

Once the regressor matrix $W$, the covariance matrix of the torque data $\Sigma$ and the torque vector $Y$ has been constructed, the condition number of $W$ was checked as well as its full rank feature. In the case of the VP-6242G Denso robot, $\vartheta$ is of dimension $(66 \times 1)$. The estimation of the parameter vector $\vartheta$ was performed by solving the CWLS optimization problem in (18). For this, we used the Matlab function fmincon that allows to find a minimum solution of the constrained nonlinear multivariable problem.

The table 4 shows two sets of numerical values of the base parameter vector $\theta_{B}$ estimated by solving the WLS problem in (10) and the CWLS one in (17) \& (18). The third column of the table presents the standard deviation $\sigma$ of the estimated parameter vector, defined as the squared root of the diagonal elements of the matrix $C$.

The standard deviation $\sigma$ of each parameter in Tab. 4 is small enough compared to the corresponding estimated value. It means that we can be confident in the parameter estimation.

The table 5 shows, for each link $i$, the virtual mass $m_{i}$ and the minimum eigenvalue of the virtual inertia tensor matrix ${ }^{i} I_{g_{i}}$. This table shows that the condition (13) is satisfied, ensuring a positive definite inertia matrix derived from the estimated parameters.

\subsection{Torque reconstruction}

Torque reconstruction is a step for checking the validity of the estimated parameters, and at the same time the corresponding dynamical model. For this, a different set of measurement than the one exploited for the identification is used. These new data were obtained by using a new input signal but with the same features as the one used for the identification 
$($ PES + periodic...). The feedback control is used with the same proportional gains. The obtained signals are different compared to those obtained during the identification. The post-processed joint torques are compared with those resulting from the application of the corresponding joint variables $(q, \dot{q}$ and $\ddot{q})$ on the dynamical model of the robot, using the identified parameters. In Fig. 4, the measured torques after filtering are compared with those calculated using the identified parameters.

The figures in Fig. 4 illustrate a good quality of reconstruction of the torques. The dynamical model of the robot and the identified parameters can be considered acceptable and used to apply control techniques.

\section{Conclusion}

In this paper, we were interested in identifying the dynamic parameters of the industrial manipulator robot Denso VP$6242 \mathrm{G}$. To this end, we used a nonlinear weighted least squares formulation of the identification problem under nonlinear constraints in the dynamic parameters. The identified parameters and the dynamic model of the robot were validated by the torque prediction approach. The identification method appeared to be efficient in giving parameters that lead to a positive definite inertia matrix. Future works will concern the use of the identified model of the Denso robot to achieve a PHRI for a co-manipulation purpose, as proposed in [Jlassi et al., 2014].
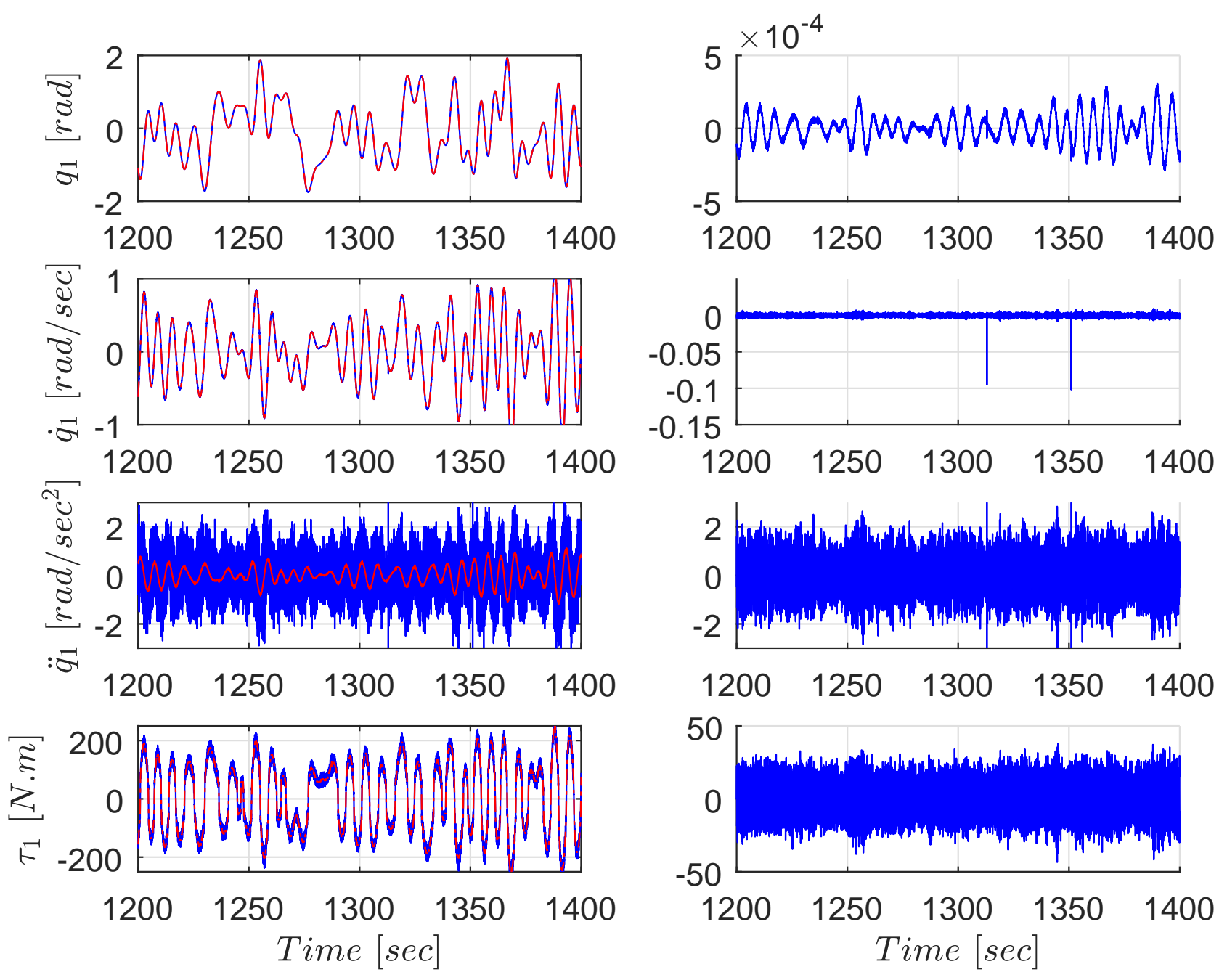

Figure 3: Data for axis 1 before (blue) and after (red) filtering (left), and the corresponding signal errors (right). 
Table 4: Numerical values of $\theta_{B}$ and standard deviation of parameters $\sigma$.

\begin{tabular}{c|c|c||c|c|c||c|c}
\hline \hline$\theta_{B}$ & CWLS & WLS & $\sigma$ & $\theta_{B}$ & CWLS & WLS & $\sigma$ \\
\hline \hline$z z r_{1}$ & 7.10 & 9.05 & $3.64 e-2$ & $y z_{4}$ & -0.06 & -0.07 & $2.83 e-3$ \\
\hline$F s_{1}$ & 49.75 & 49.38 & $7.68 e-3$ & $z z r_{4}$ & 0.06 & -0.86 & $5.71 e-3$ \\
\hline$F v_{1}$ & 150.81 & 151.39 & $2.78 e-2$ & $m x_{4}$ & 0.30 & 0.24 & $7.84 e-4$ \\
\hline$x x r_{2}$ & -5.96 & 0.20 & $4.46 e-2$ & $m y r_{4}$ & 0.20 & 0.20 & $6.08 e-4$ \\
\hline$x y_{2}$ & -1.01 & -1.22 & $2.15 e-2$ & $F s_{4}$ & 24.63 & 24.62 & $4.75 e-3$ \\
\hline$x z r_{2}$ & -1.17 & -1 & $1.35 e-2$ & $F v_{4}$ & 21.55 & 21.17 & $1.13 e-2$ \\
\hline$y z_{2}$ & -0.97 & -0.81 & $2.19 e-2$ & $x x r_{5}$ & 0.22 & 0.29 & $4.87 e-3$ \\
\hline$z z r_{2}$ & 17.29 & 17.71 & $2.56 e-2$ & $x y_{5}$ & 0.01 & 0.013 & $2.42 e-3$ \\
\hline$m x r_{2}$ & 18.67 & 18.77 & $2.54 e-3$ & $x z_{5}$ & -0.02 & -0.01 & $1.73 e-3$ \\
\hline$m y_{2}$ & 0.28 & 0.31 & $1.22 e-3$ & $y z_{5}$ & 0.01 & 0.01 & $2.06 e-3$ \\
\hline$F s_{2}$ & 63.64 & 64.61 & $1.12 e-2$ & $z z r_{5}$ & 0.56 & 0.59 & $1.96 e-3$ \\
\hline$F v_{2}$ & 185.75 & 180.34 & $4.80 e-2$ & $m x_{5}$ & 0.13 & 0.13 & $4.58 e-4$ \\
\hline$x x r_{3}$ & 9.04 & -0.32 & $2.89 e-2$ & $m y r_{5}$ & 2.12 & 2.11 & $4.79 e-4$ \\
\hline$x y r_{3}$ & 0.41 & 2.27 & $1.91 e-2$ & $F s_{5}$ & 20.45 & 20.40 & $3.79 e-3$ \\
\hline$x z_{3}$ & 0.05 & 0.10 & $5.68 e-3$ & $F v_{5}$ & 29.29 & 29.12 & $9.64 e-3$ \\
\hline$y z_{3}$ & 0.04 & 0.04 & $5.97 e-3$ & $x x r_{6}$ & -0.07 & -0.08 & $2.34 e-3$ \\
\hline$z z r_{3}$ & 4.37 & 4.20 & $4.14 e-3$ & $x y_{6}$ & 0.03 & 0.03 & $1.24 e-3$ \\
\hline$m x r_{3}$ & -5.16 & -4.98 & $1.24 e-3$ & $x z_{6}$ & 0.02 & 0.02 & $1.27 e-3$ \\
\hline$m y r_{3}$ & 8.77 & 8.79 & $1.30 e-3$ & $y z_{6}$ & 0.00 & 0.00 & $1.36 e-3$ \\
\hline$F s_{3}$ & 54.78 & 54.87 & $8.10 e-3$ & $z z_{6}$ & 0.00 & 0.05 & $2.29 e-3$ \\
\hline$F v_{3}$ & 87.92 & 86.78 & $2.95 e-2$ & $m x_{6}$ & 0.10 & 0.10 & $4.04 e-4$ \\
\hline$x x r_{4}$ & -0.21 & 0.13 & $7.32 e-3$ & $m y_{6}$ & -0.05 & -0.05 & $3.51 e-4$ \\
\hline$x y_{4}$ & 0.19 & 0.19 & $3.34 e-3$ & $F s_{6}$ & 19.08 & 19.11 & $3.91 e-3$ \\
\hline$x z_{4}$ & 0.23 & 0.21 & $2.81 e-3$ & $F v_{6}$ & 15.74 & 15.51 & $7.91 e-3$ \\
\hline \hline
\end{tabular}

Table 5: Checking of the constraints in (13).

\begin{tabular}{c|c|c|c|c|c|c}
\hline \hline Link & 1 & 2 & 3 & 4 & 5 & 6 \\
\hline \hline$\lambda_{\min }\left\{{ }^{i} I_{g_{i}}\right\}$ & 0.01 & 0.01 & 0.01 & 0.01 & 0.01 & 0.01 \\
\hline$m_{i}$ & 6.22 & 2.07 & 17.99 & 20.95 & 1.93 & 38.45 \\
\hline \hline
\end{tabular}

\section{References}

[An et al., 1985] An, C. H., Atkeson, C. G., and Hollerbach, J. M. (1985). Estimation of inertial parameters of rigid body links of manipulators. In Proc. of the IEEE Conf. on Dec. and Ctrl. (CDC), pages 990-995.

[Brunot et al., 2017] Brunot, M., Janot, A., Carillo, F., and Garnier, H. (2017). A pragmatic and systematic statistical analysis for identification of industrial robots. In Proc. of the IEEE Int. Conf. on Advanced Intelligent Mechatronics (AIM), pages 559-564, Munich, Germany. 

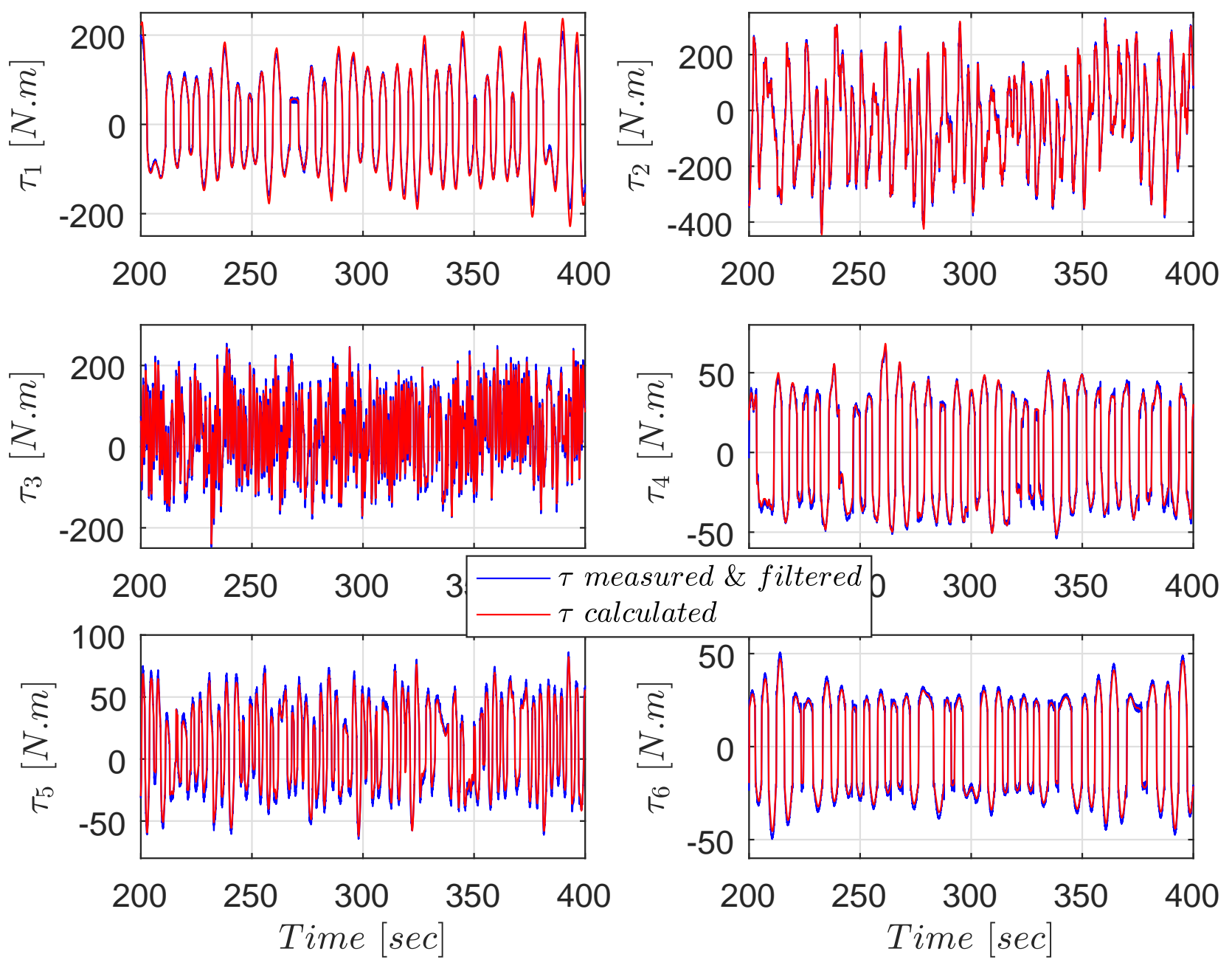

Figure 4: The filtered measured and computed torques.

[Gautier, 1990] Gautier, M. (1990). Numerical calculation of the base inertial parameters of robots. In Proc. of the IEEE Int. Conf. on Robotics and Automation (ICRA), pages 1020-1025.

[Gautier and Khalil, 1988] Gautier, M. and Khalil, W. (1988). On the identification of the inertial parameters of robots. In Proc. of the IEEE Conf. on Dec. and Ctrl. (CDC), pages 2264-2269.

[Gautier and Khalil, 1990] Gautier, M. and Khalil, W. (1990). Direct calculation of minimum set of inertial parameters of serial robots. IEEE Trans. on Robotics and Automation, pages 368-373.

[Gautier and Khalil, 1991] Gautier, M. and Khalil, W. (1991). Exciting trajectories for the identification of base inertial parameters of robots. In Proc. of the IEEE Conf. on Dec. and Ctrl. (CDC), pages 494-499.

[Janot et al., 2014] Janot, A., Vandanjon, P.-O., and Gautier, M. (2014). A generic instrumental variable approach for industrial robot identification. IEEE Trans. on Ctrl. Syst. Tech., 22(1):132-145.

[Jin and Gans, 2015] Jin, J. and Gans, N. (2015). Parameter identification for industrial robots with a fast and robust trajectory design approach. Robotics and Computer-Integrated Manufacturing, 31:21-29.

[Jlassi et al., 2014] Jlassi, S., Tliba, S., and Chitour, Y. (2014). An event-controlled online trajectory generator based on the human-robot interaction force processing. Industrial Robot: An International Journal, 41:15-25. 
[Khalil and Creusot, 1997] Khalil, W. and Creusot, D. (1997). Symoro+: A system for the symbolic modelling of robots. Robotica, Cambridge University Press, pages 153-161.

[Khalil and Kleinfinger, 1986] Khalil, W. and Kleinfinger, J.-F. (1986). A new geometric notation for open and closedloop robots. In Proc. of the IEEE Inter. Conf. on Robotics and Automation (ICRA), pages 1174-1179.

[Khalil et al., 2014] Khalil, W., Vijayalingam, A., Khomutenko, B., Mukhanov, I., Lemoine, P., and Ecorchard, G. (2014). Opensymoro: An open-source software package for symbolic modelling of robots. IEEE/ASME Int. Conf. on Advanced Intelligent Mechatronics (AIM), pages 1206-1211.

[Khosla and Kanade, 1985] Khosla, P. K. and Kanade, T. (1985). Parameter identification of robot dynamics. In Proc. of the IEEE Conf. on Dec. and Ctrl. (CDC), pages 1754-1760.

[Lennart, 1971] Lennart, L. (1971). Characterization of the concept of 'persistently exciting' in the frequency domain. Technical report, Department of Automatic Control, Lund Institute of Technology (LTH).

[Swevers et al., 2007] Swevers, J., Verdonck, W., and Schutter, J. D. (2007). Dynamic model identification for industrial robots. IEEE Control Systems Magazine, 27(5):58-71.

[Walter and Pronzato, 1997] Walter, E. and Pronzato, L. (1997). Identification of Parametric Models from Experimetal Data. Communications and Control Engineering. Springer-Verlag London, 1 edition.

[Yoshida and Khalil, 2000] Yoshida, K. and Khalil, W. (2000). Verification of the positive definiteness of the inertial matrix of manipulators using base inertial parameters. Int. Journ. of Rob. Res., 19(5):498-510. 